\title{
Virtual reality environment to study the ergonomic features of firefighters' activity
}

\author{
Tadeusz Nowicki ${ }^{1,},{ }^{*}$ Jarosław Koszela ${ }^{1}$ and Lukasz Matuszelański ${ }^{1}$ \\ ${ }^{1}$ Military University of Technology, Faculty of Cybernetics, Urbanowicza 2, 00-908 Warsaw, Poland
}

\begin{abstract}
This work considers the problem of studying the strategy of equipment location on the firefighting vehicle. A unified firefighter's vehicle Euro Truck is being designed. The problem of location the emergency equipment on the vehicle is very important from the point of view of firefighters ergonomic. It is consider two ergonomic criterion functions: how long is the distance of horizontal and vertical displacement of equipment during its unloading. The third ergonomic criterion is optimization of firefighters work time. These criteria lead to a reduction in the physical load of firefighters' bodies. Computer simulation, including virtual reality, allows for a very detailed study of the work of firefighters during the removal of equipment from the vehicle. A simulation environment with virtual reality was built and tested in order to measure ergonomic criteria during firefighters' work in a rescue operation for various action scenarios.
\end{abstract}

\section{Introduction}

In recent years, the phenomenon of virtual reality (VR) is reflected in millions of people who are completely absorbed in computer games. Simulation models are used in various types of applications $[1,2,3]$. Simulators should have high reliability [4]. An enormous flow of data during simulation has to be analyzed $[5,6,7]$.

The performance of modern personal computers allows to build increasingly more graphically advanced products. The powerful processing capacities of GPUs create a virtual world with a level of detail similar to the one that sees the human eye in reality. Therefore, the virtual reality should be understood as the simulation of fragments of the world in which we live every day or the creation of completely invented worlds. This simulation is mainly experienced by two out of five senses: sight and sound. The simplest form of virtual reality is a threedimensional image that can be interactively explored on a computer using the keyboard and mouse in such a way that the content of the image moves in the selected direction, enlarged or reduced. The three-dimensional world created by modern computers makes people feel present in it both mentally and physically, thinks that they live in a completely credible virtual world. What distinguishes VR from ordinary computer use is the nature of input and output devices. While working in a conventional manner on the computer we use keyboard, mouse and sometimes speech recognition devices so immersed in virtual reality, we have a large collection of sensors detecting the movement of the human body, steering wheels, manipulators, controllers in the form of replica weapons and other such devices, which are supposed to transfer a more real impression to these digital ones. Similarly, things happen when displaying output data, in a traditional computer use we use a screen or sometimes a printer. The VR system, in contrast, uses: goggles (double screens - one for each eye), curved large-scale screens, gyroscopic platforms and other dedicated output devices that increase the feelings.

\section{Elements of VR environment}

The most common VR input and output devices are:

- Head-mounted displays (HMD) - helmets, goggles, or cardboard frames with built-in lenses converting a regular smartphone into a primitive HMD. These devices are designed to display a 3D image that changes smoothly in real time with the movement of the head. Two displays located one in front of each eye are surrounded by a blackout curtain that eliminates all other images and lights from the real world (Fig.1). Two screens also display slightly different stereoscopic images thus creating a realistic $3 \mathrm{D}$ perspective of the virtual world. HMD devices usually have built-in accelerometers or position sensors, so they accurately detect head and body movements, including position and orientation - the way they should tilt.

\footnotetext{
* Corresponding author: tadeusz.nowicki@,wat.edu.pl
} 


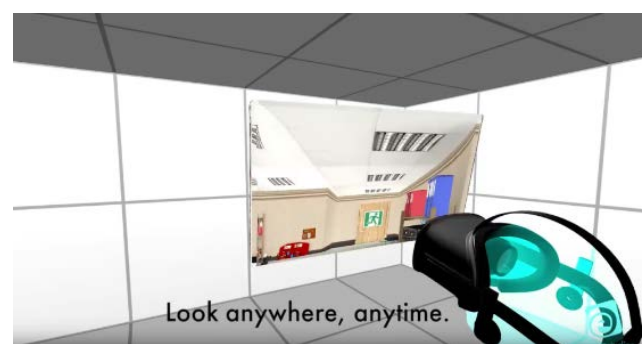

Fig. 1. The way the head mounted display device works.

- Immersive rooms - rooms on walls that display smoothly changing images when moving. The technique of displaying virtual reality images is used, among others, in flight simulators, in which images of landscapes, cities and approaches to airport plates are displayed on large screens surrounded by an airplane cockpit (Fig.2).

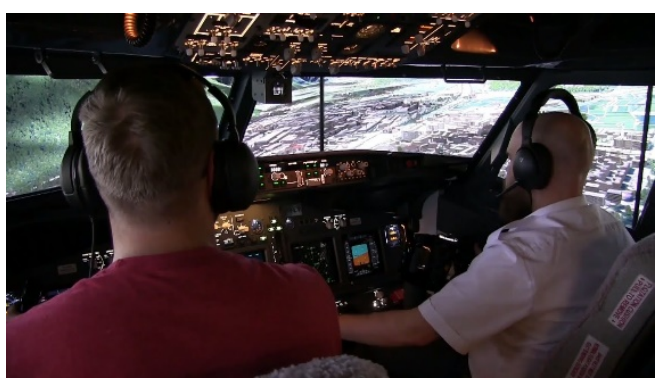

Fig. 2. Boeing 737 simulator.

- Haptic gloves - gloves with sensors to detect the movement of hands and figures. This type of virtual reality controller allows you to deal with VR objects in a way related to natural instinct. Seeing a new object or wanting to explore the already known one, we do it by hand and touch. From a technical point of view, fiber optic cables stretched over the length of each finger are used in haptic gloves. Each of the cables has small incisions on it which, while the finger is bent, emit more or less light on the outside of the cable. The photocell at the end of the cable measures the amount of light that goes into it and on this basis the computer determines the position of the finger. Other gloves use strain gauges, piezoelectric sensors or electro-mechanical devices such as potentiometers to measure finger movements. Recently, work has been underway on haptic coupling in such gloves, thanks to which you can feel the size and shape of the object held in your hand. On the glove, air-filled cushions are placed, and then their hardness is controlled, expanded or compressed to reflect the illusion that a virtual object is in the hand (Fig.3).

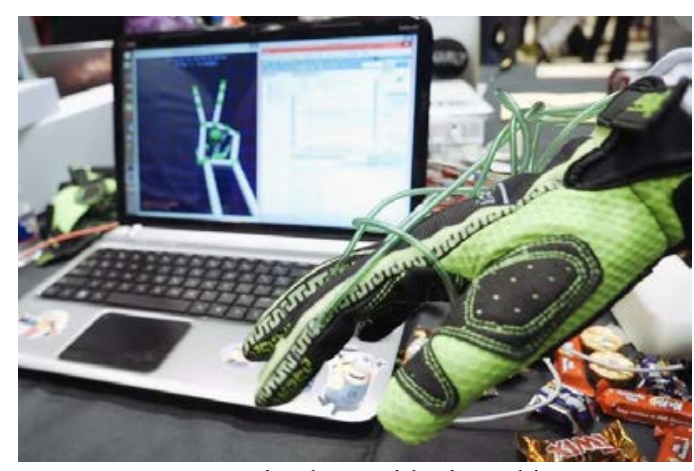

Fig. 3. Haptic glove with air cushions.

\section{Examples of the use of virtual reality}

Immersiveness, or the process of immersing into an electronic reality is a new technological trend that allows you to look at various issues from a perspective that has hitherto been unattainable. VR is applicable in many areas of life.

Education and training, in which using virtual reality you can simulate situations encountered while performing difficult and dangerous jobs (Fig.4). Training emergency services in real conditions can pose a threat to life or health, which VR avoids. Training to perform professions that can be limited by the availability of specialized machines on a daily basis, using virtual reality can be implemented without these limitations and at lower costs.

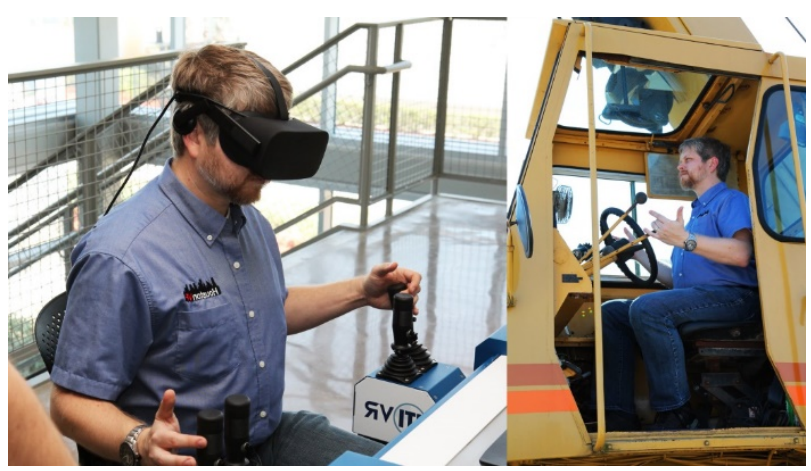

Fig. 4. Training for crane construction operator.

Medicine, in which, in addition to surgical training on virtual organs of the patient corresponding to this real VR, it also enables telemedicine, i.e. testing and operating patients remotely. From a technical point of view, the distance operation is that the surgeon is connected in one place to the control panel in virtual reality, while the robot holding the proverbial surgical knife is located elsewhere, it can even be on another continent. The best known example of such remote surgical operations is the daVinci surgical robot (Fig.5). The use of VR in the field of telemedicine during particularly complex operations gives the opportunity to cooperate with the group of the best surgeons in the world. 


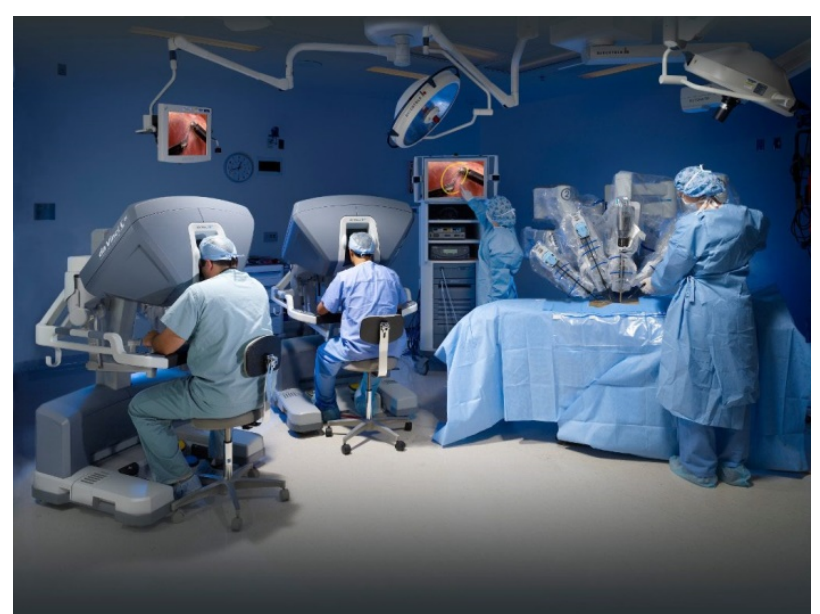

Fig. 5. Operation using VR - daVinci robot.

Simulator KTO Rosomak SK-1 Pluton, made in Cybernetics Faculty of Military Univesrsity of Technology, is a training simulator operating in virtual reality, in which the main emphasis was placed on the realism of mapping the positions of the crew of a wheeled Rosomak armored personnel carrier. The gunner and the commander entering the tower cabin in the simulator have a sense of exactly the same space that is in the real vehicle. At hand, they have a full set of switches, buttons and keypads located in places corresponding to those in a physical vehicle. On dedicated screens and periscopes, images from the virtual battlefield are displayed, on which the crew of KTO Rosomak are trained. The tower's cabin is closed, which further increases the VR experience. The crew, when launching and preparing the virtual tower model for combat operations, must perform exactly the same sequence of key presses and releases as in the real vehicle. When shooting, bullets in VR feature exactly the same ballistics as in actual conditions. The cab of the driver's seat is additionally equipped with a gyroscopic platform, which transmits the vibrations experienced by the driver while driving over uneven terrain in computer reality. The simulator is designed to teach the service of the actual vehicle by learning how to use it in virtual reality (Fig.6).

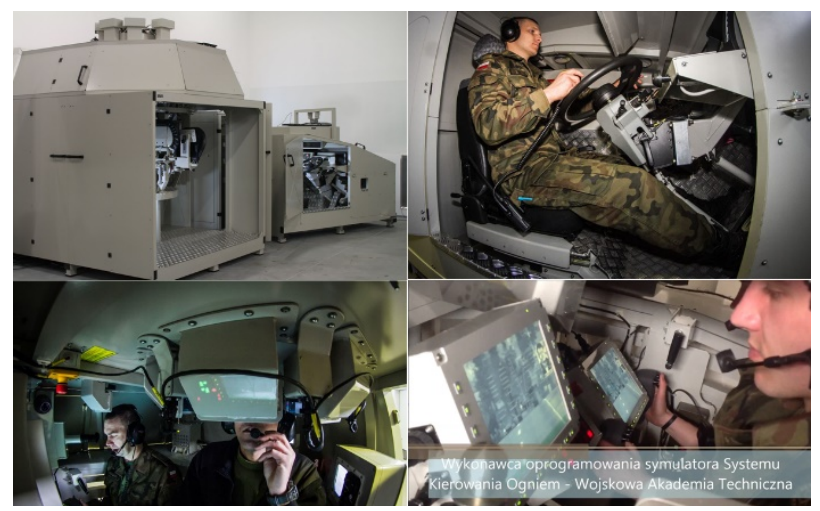

Fig. 6. Simulator KTO Rosomak SK-1 Pluton.

\section{Ergonomics in the ErgoTruck fire vehicle}

In this work, the problem of deploying firemen' equipment is discussed in order to achieve the best value of ergonomic indicators for fireman during anti-fire action. The equipment can be located on the fire truck Ergo Truck in a different ways. We are looking for the best way to do it from the point of view of ergonomics. The multicriterial optimization problem of equipment location on the vehicle has been formulated. It is linear integer programming problem with three criteria functions. It is able to solve that problem using formal method after some modifications [8]. It is possible to receive several solutions from multicriterial optimization problem and then evaluate them in a virtual simulation environment. It can then be taken into account a number of additional factors that are important for fireman and ergonomics.

The development of standards, equipment and activities to optimize the working conditions is to determine the equipment to be deployed in the rescue and fire fighting vehicle and to deploy it in the useable recesses of rescue and firefighting vehicles designed for this purpose.

The result of this study are the guidelines for designers equipping new or upgraded fire trucks. In the issue of deployment of equipment in firefighting vehicles are guided by the working conditions of persons - the personnel of the vehicle. A number of factors are taken into account, but the problem is primarily dedicated to take into account ergonomic factors. It should be emphasized that these factors are particularly important in multiple actions in a relatively short time and refer to the same group of people. Possible effects of misplaced equipment in firefighting vehicle will be cumulatively accumulated to the health of the crew members of the vehicle. The definition of criteria for evaluating working conditions is mainly related to the ergonomics of firefighters taking part in rescue operations.

The problem of empirical study of human work in concrete conditions of material work environment is not easy to solve in a short time. It would be necessary to carry out a number of specialized personnel surveys for various equipment installations in firefighting vehicles in a large sample of various types of firefighting operations. This would not only be a long-term undertaking but would also add to the health of the staff. So decided to solve quite innovative. Computer simulations of firefighting per-sonnel actions for various types of actions will be used to simulate the various deployment variants on the vehicle in terms of ergonomics.

The ErgoTruck simulator is designed to map the actions of the crew of the rescue and fire-fighting vehicle for a particular type of action with different variants of the arrangement of the equipment in the vehicle dedicated to ergonomics. In addition to high graphical details in the virtual models of equipment, firefighters and rescue and fire fighting vehicle, the level of VR realism created in the simulator increases the use of Oculus Rift goggles. 
The VBS system supports this type of device. After putting on the goggles and starting the simulation, and then calibrating the Oculus position by a one-touch keyboard shortcut, the goggles position on the user's head is synchronized with the virtual character's head controlled by the user in the simulation.

From that moment on, with the help of head movements, he has the fluent opportunity to view the computer world around him in the 360 degrees around his axis. Sitting in the cabin of the rescue and firefighting vehicle, the user can turn the head of the vehicle to the left and right, while leaning forward, can approach the vehicle's windshield in virtual reality and look behind it to see what is directly in front of the vehicle's hood.

Getting out of the vehicle and being in the immediate vicinity of running firefighters, the user directing his figure with the help of head movements and additional control of movement with the controller has the feeling of moving among the firemen running near him and carrying equipment. Moving the user in virtual reality generates additional difficulties for the crew unloading the equipment, I have to avoid the person standing in the way. The creation of the Oculus Rift goggles during the simulator operation adds realism to the virtual reality, moreover, it is more practical from the point of view of a more intense tracking of individual stages of the computer simulation.

As a result of these considerations we have the situation that rescue and firefighting equipment can be placed in recesses on both sides of the vehicle. But these are not many. The following drawings (Fig.7) show the recesses that may be considered in the deployment of rescue and firefighting equipment on the sides of the rescue and fire fighting vehicle.

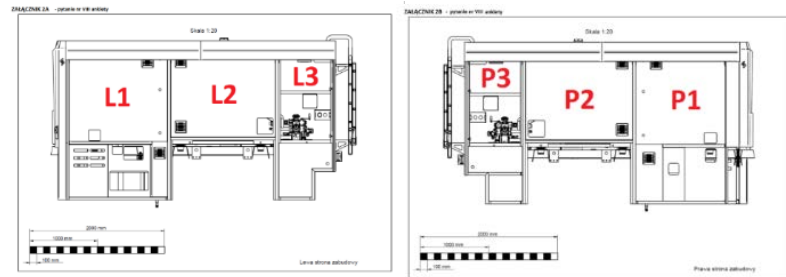

Fig. 7. ErgoTruck luggage spaces.

\section{Simulator ErgoTruck}

Simulator ErgoTruck was constructed in VBS3 environment [3]. VBS3 (Virtual Battlespace 3) is a highresolution virtual simulation environment that has been made and developed by Bohemia Interactive Simulations (BISim).

The software included in the simulator was written entirely using the SQF scripting language characteristic of the VBS3 virtual programming environment. The simula-tion module is responsible for the course of the individual simulation experiments. It consists of modules: placement, concurrent transfer and analytical. The deployment module uses the data provided by the simulation parameter configuration module, primarily from data structures powered by information from text files that include methods for deploying rescue and firefighting equipment. Each rescue and firefighting equipment is identified by the P3D model class name and has appropriate visualization in the VBS3 simulation environment. Models of examples of rescue and firefighting equipment are shown on the figure Fig. 8 .

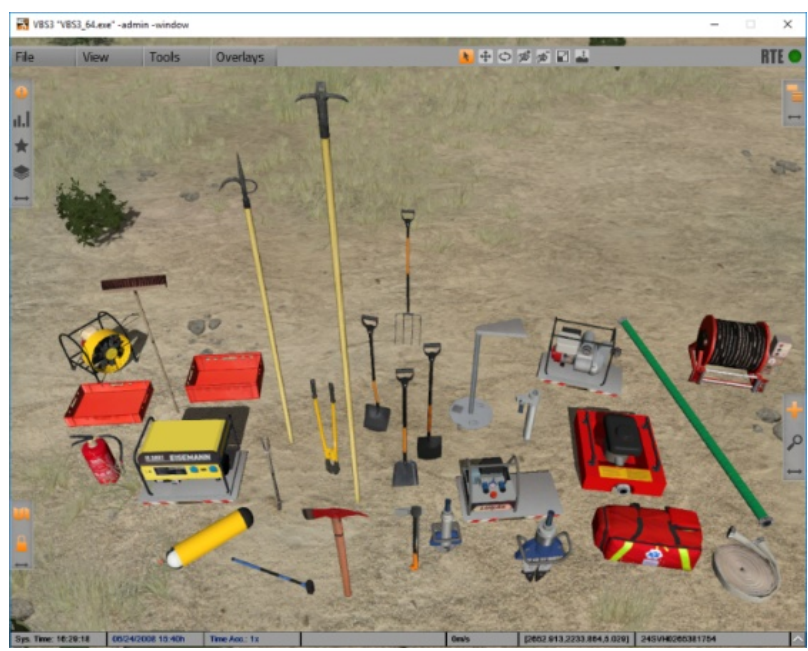

Fig. 8. ErgoTruck equipment examples.

The rescue and firefighting equipment resulting from the deployment module is used in the other two modules included in the simulation module. The concurrent transfer module also uses the data provided by the simulation parameter configuration module. Within this module SQF scripting tools have been implemented with appropriate co-programming capabilities to simulate the transfer of rescue and firefighting equipment from the luggage space to the place of location by a specific number of firefighters. The transfer schedule is strictly dependent on the information received by the input integration module from the corresponding configuration file with the transfer sequence. The weight of individual hardware objects determines the speed of firefighting by the fireman's AI, and in addition, some models require two firefighters to transfer simultaneously, resulting in simulated stopping of the appropriate firefighters AI while waiting for the firefighter to complement the wearer pair.

The analytical module uses the results of the deployment modules and the concurrent transfer and enumerates some statistics gathered during simulation. The method of calculating statistics is based on AI algorithms, such as avoiding collisions of firefighters in traffic and calculating alternative traffic routes in the event of a collision threat. Received statistics are sent out to the reporting module. The analytical module collects the data from which luggage space was transferred to the rescue and firefighting equipment to which the height was raised, how far it was moved, during which time the firefighter was, etc. In addition, the analytical module manages the thread responsible for the elapsed simulation time. The report module assumes data provided by the simulation module, and groups them accordingly and places them in the output files of the CSV. Report files include the results of simulated experiments performed by individual rescue and firefighting equipment. For each row identified by the 
object, the specific class name of its P3D model is assigned attributes such as: weight of the equipment, number of persons required to move it, length of access roads to the equipment, length of its lifts, lifting and simulation times for starting and ending work. The main modules that make up the software with the dependency between them are shown on the figure 9 .

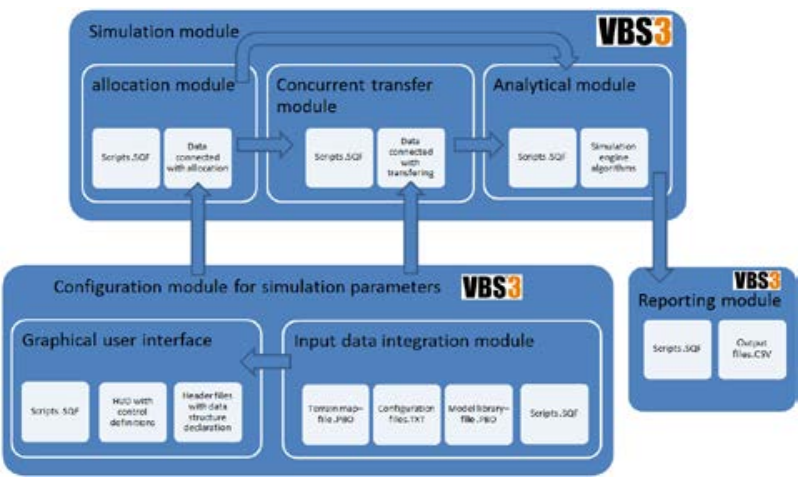

Fig. 9. ErgoTruck simulator modules.

On figure Fig. 10 we can see possibly equipment location in fire truck recesses. In scenarios prepared for simulation experiments many possible allocation of equipment is considered. We try to check which one is the best.

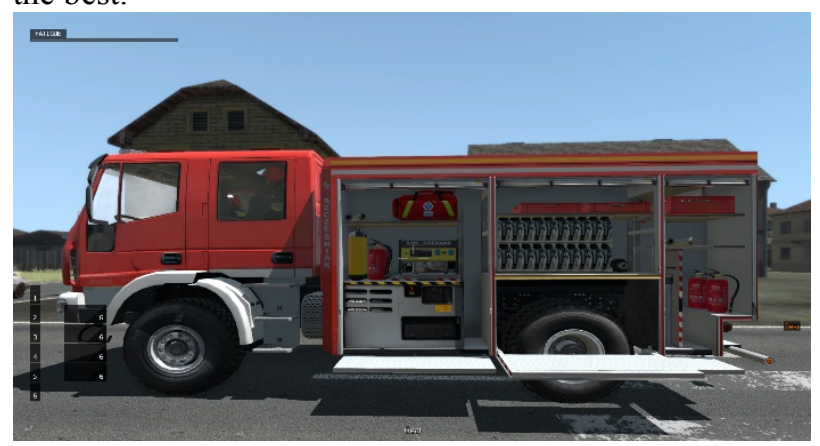

Fig. 10. Distribution of rescue and firefighting equipment on fire truck.

On figure Fig.11 we see firemen start to remove equipment from fire trucks. In our simulator all firefighters' movements are modeled to be closest to their natural activities.

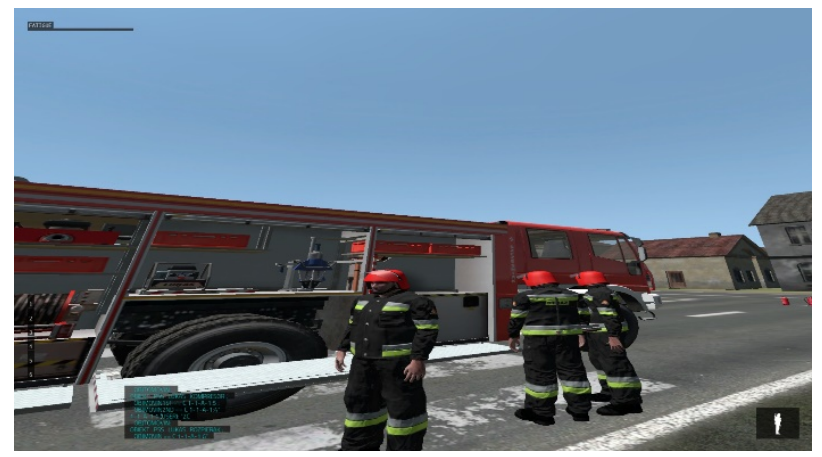

Fig. 11. Simulation of moving equipment in the order of schedule.

On figure Fig.12 and figure Fig.13 it is shown how firemen take individual pieces of equipment and transfer them to fixed locations. There may be several different places for locating equipment.

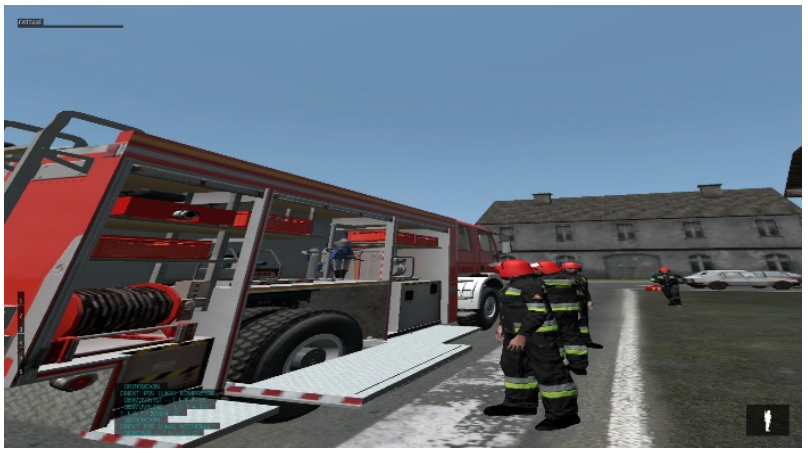

Fig. 12. Firemen start taking individual pieces of equipment.

The arrangement of emergency equipment elements may be different for many scenarios of simulation experiments. Depending on the location of the equipment on the vehicle, the effort of firefighters is different. The arrangement of emergency equipment should be selected so that the firemen's effort at work is as small as possible. Different scenarios serve this purpose. They allow us to choose the optimal location from an ergonomic point of view $[9,10,11]$. They collected at the numerous information about the activities of people $[10,12]$.

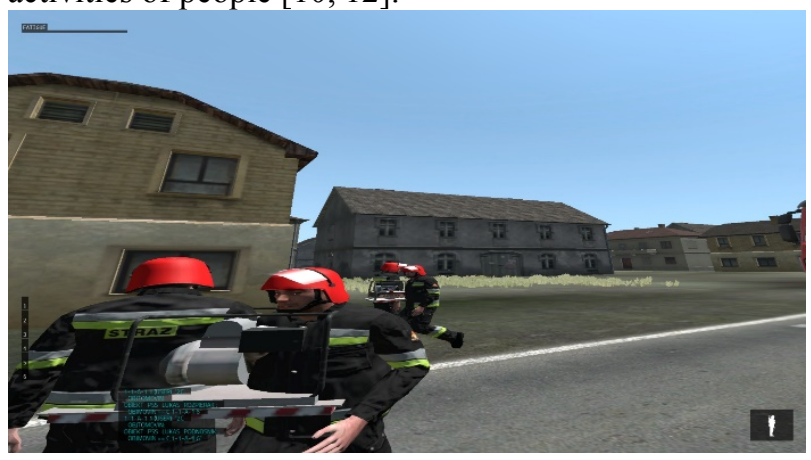

Fig. 13. Firemen carry rescue equipment.

The place of folding the rescue equipment is established. Firefighters are to move rescue equipment from fire vehicle to a designated place (Fig.14.). Their movement is simulated while moving the equipment.

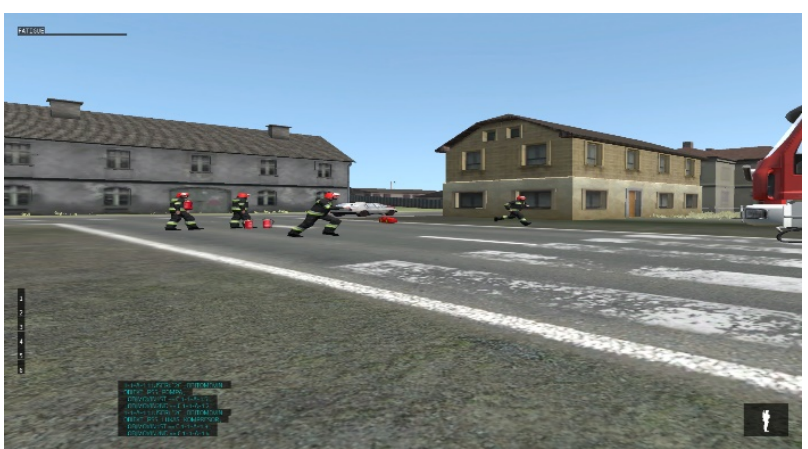

Fig. 14. Firemen carry equipment to destination place.

At the same time, the horizontal and vertical displacement of the equipment and route of moving the equipment by firemen are measured (Fig.15, Fig.16, Fig. 
17). A number of factors are taken into account, but the problem is primarily dedicated to take into account ergonomic factors. It should be emphasized that these factors are particularly important in multiple actions in a relatively short time and refer to the same group of people. Possible effects of misplaced equipment in firefighting vehicle will be cumulatively accumulated to the health of the crew members of the vehicle. The definition of criteria for evaluating working conditions is mainly related to the ergonomics of firefighters taking part in rescue operations.

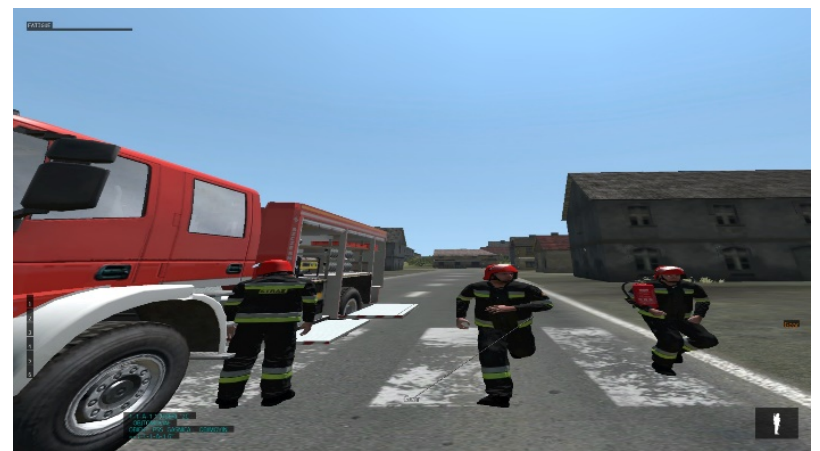

Fig. 15. Firemen start carry rescue equipment.

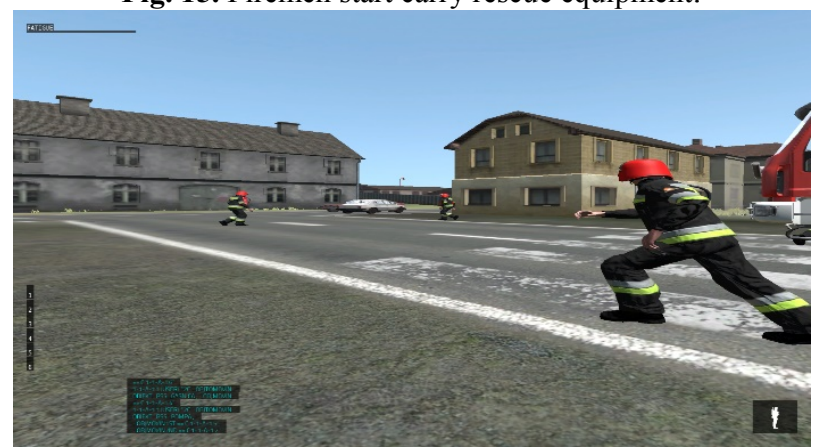

Fig. 16. Firemen are continuing to move the equipment.

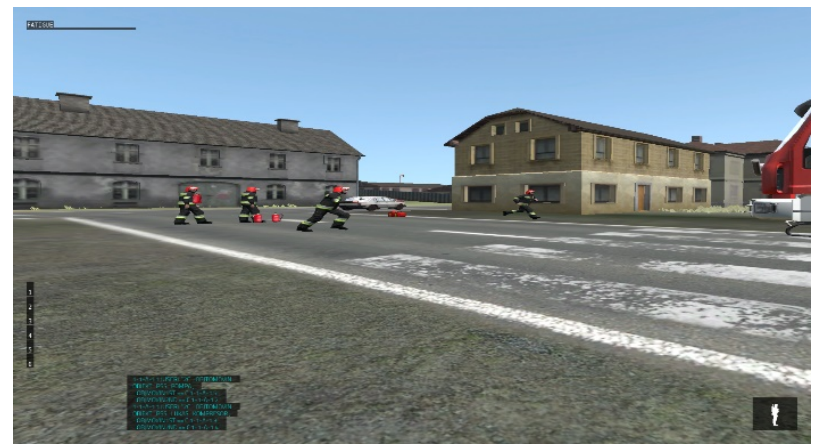

Fig. 17. Firemen finish moving rescue equipment.

At the end of the simulation, the rescue equipment is laid out by the firefighters in a fixed place (Fig.18). Then the firefighters' action ends.

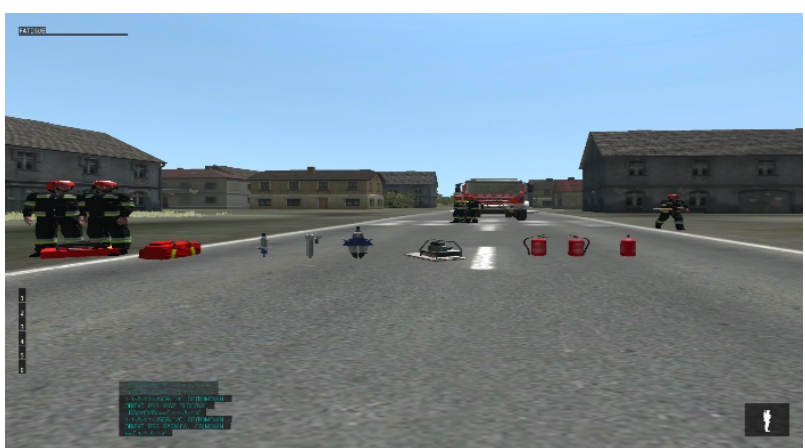

Fig. 18. Rescue equipment is laid out by the firefighters in a fixed place.

During the virtual simulation, firefighters can be observed from inside the fire vehicle (Fig19).

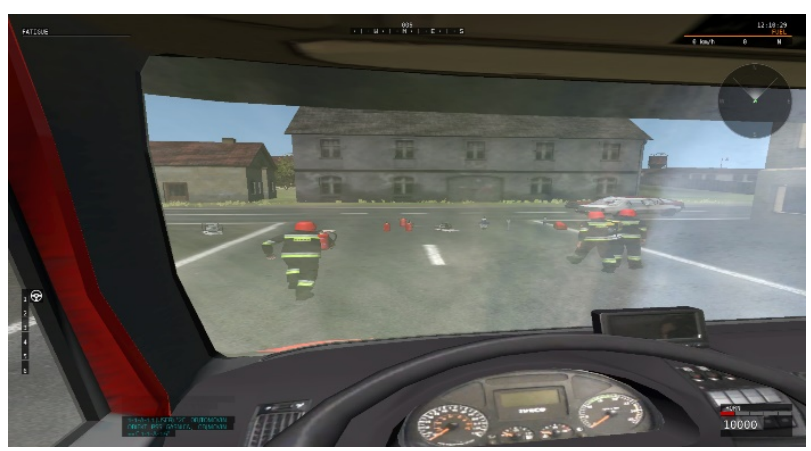

Fig. 19. Rescue equipment is laid out by the firefighters in a fixed place.

Also from the inside of a fire truck you can see the situation when firefighters have already completed their action. All equipment items are already located in the right place. Firefighters stand with equipment folded on the ground (Fig.20).

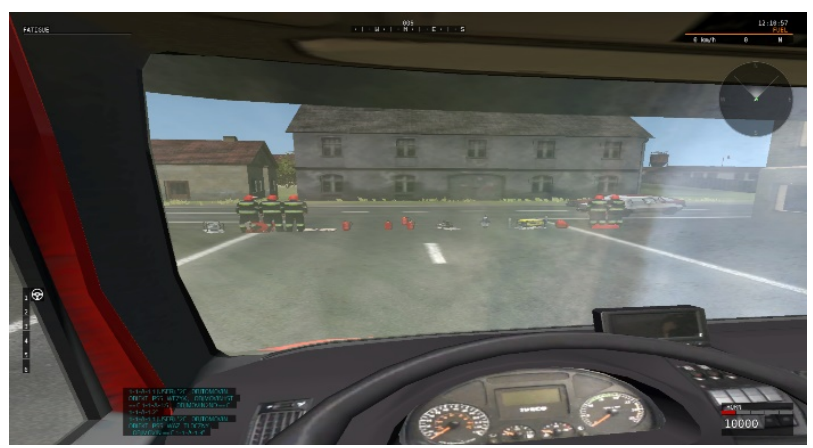

Fig. 20. View of stacked rescue equipment from the fire engine's cabin.

The final simulation report includes:

- name of equipment element,

- code of equipment element,

- number of fireman,

- length of every equipment element way to final location,

- the moment when the element starts moving,

- the moment of completion of displacement of the element,

- and many other characteristics.

The form of the final report with simulation results is shown on figure Fig. 21 given below. 


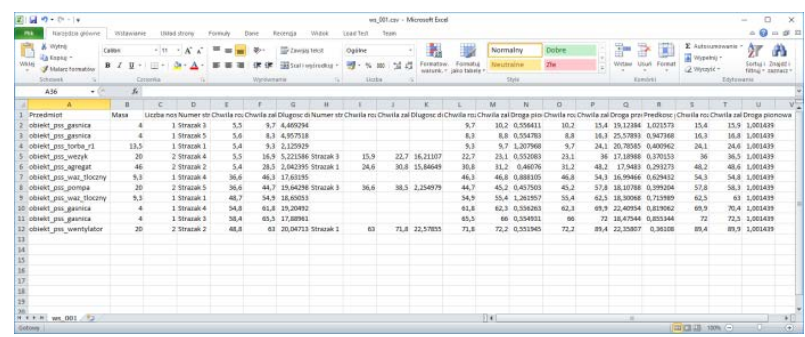

Fig. 21. Content file with simulation results.

\section{Conclusions}

In this paper there are presented examples of using virtual simulators to build trainers demonstrate their great possibilities in this regard. The main issue pursued by the engines of virtual simulators is to simulate the phenomena occurring in the virtual world with regard to the conditions existing in the real world.

VBS3 simulation environment along with a set of additional tools provides a comprehensive solution for supporting serious exercises like firemen's actions.

The trainers are focused on the training of individuals, groups, task forces, holders of positions and equipment operators. Such properties of virtual simulation environments allow reproducing a high level of immersion of conducted computer-assisted exercises, which is an important element of exercises carried out using them.

The work presents project and implementation of a virtual simulator for the ergonomic study of the firefighter's performance at the time of removal of firemen equipment from fire truck. It is extremely important for firefighters to have as little workload as possible during their work. Firefighters must remove firefighting equip-ment in a short time and causing high physical load for their bodies. Virtual simulation will allow to measure the physical loads of firemen' bodies in different types of actions. The basic modules of the simulator are shown and discussed. Several pictures from simulator interface allow us to show how simulation experiments are running. Many of the characteristics needed to determine the correct placement of equipment on a fire truck can be measured during the time of the simulation expedition.

\section{Acknowledgements}

This work was carried out as part of the project. DOB-BIO7/07/02/2015 ERGO TRUCK financed by EU.

\section{References}

1. J. Gregory, Game Engine Architecture - second edition (CRC Press, ISBN-13: 978-1-4665-6006-2, Les Ulis, ( 2015)

2. G. C. Burdea, P. Coiffet, Virtual Reality Technology (Wiley-IEEE Press, ISBN: 978-0-4713-6089-6, Hoboken, 2003) J. Gregory, Game Engine Architecture - second edition (CRC Press, ISBN-13: 978-1-4665-6006-2, Les Ulis, (2015)
3. J. Koszela, R. Wantoch-Rekowski, Designing advanced vehicle trainers using virtual simulation technology, Journal of KONES, 22, pp. 265-272, (2015)

4. R. Waszkowski, M. Kiedrowicz, T. Nowicki, Z. Wesołowski, K. Worwa, Method for assessing software reliability of the document management system using the RFID technology, MATEC Web of Conferences, vol. 76, DOI: 10.1051/matecconf/ 20167604009 Greece, (2016)

5. R. Waszkowski, M. Kiedrowicz, T. Nowicki, K. Worwa, Customer service processes automation in administrative office with RFID tagged documents, Fourth International Conference on Mathematics and Computers in Sciences and in Industry, pp. 224-233, (2017)

6. M. Kiedrowicz, T. Nowicki, R. Waszkowski, Business Process Data Flow between Automated and Human Tasks, Proceedings of the 3rd International Conference on Social Science (ICSS 2016), vol. 1, ISBN: 978-1-60595-410-3, Shanghai, China, (2016)

7. M. Kiedrowicz, T. Nowicki, R. Waszkowski, Z. Wesołowski, K. Worwa, Software simulator for property investigation of document management system with RFID tags, MATEC Web of Conferences, vol. 76, DOI: 10.1051/matecconf/20167604012, Greece, (2016)

8. M. Kiedrowicz, T. Nowicki, R. Waszkowski, Z. Wesołowski, K. Worwa, Optimization of the Document Placement in the RFID Cabinet, EDP Science, MATEC Web of Conferences 76 02001, 2016, DOI:10.1051/matecconf/20167602001, (2016)

9. M. Jasiulewicz-Kaczmarek, A. Saniuk, T. Nowicki, The maintenance management in the macroergonomics context, Advances in Social \& Occupational Ergonomics vol 487, Springer Series, Advances in Intelligent Systems and Computing, Goossens R.H.M. (Ed.), pp. 35-46, (2017)

10. T. Nowicki, R. Waszkowski, A. Saniuk, HumanComputer interaction in sanitary inspection simulation exercises, Advances in Ergonomics Modeling, Usability \& Special Populations, in series: Advances in Intelligent Systems and Computing, vol. 486, pp. 245-254, Springer International Publishing AG, 2017W. van der Aalst, M. van H. Kees, Workflow Management, Models, Methods and Systems, The MIT Press Cambridge, (2002)

11. Saniuk A., Saniuk S., Cagáňová D., Čambál M., Control of strategy realization in metallurgical production, 23th International Conference on Metallurgy and Materials - METAL 2014, TANGER, Czech Republic, Brno, pp. 1876-1881, (2014)

12. P. Raulefs, S. Shoukourian, L. Tarumyan, V Matevosyan, Determination of critical paths in hammock type processes, HPC'2003, SCS International Advanced Simulation Technologies Conference ASTC'2003, p. 241-246, (2003) 